\title{
In vitro histone lysine methylation by NSD1, NSD2/MMSET/WHSC1 and NSD3/WHSC1L
}

\author{
Masayo Morishita, Damiaan Mevius and Eric di Luccio*
}

\begin{abstract}
Background: Histone lysine methylation has a pivotal role in regulating the chromatin. Histone modifiers, including histone methyl transferases (HMTases), have clear roles in human carcinogenesis but the extent of their functions and regulation are not well understood. The NSD family of HMTases comprised of three members (NSD1, NSD2/ MMSET/WHSC1, and NSD3/WHSC1L) are oncogenes aberrantly expressed in several cancers, suggesting their potential to serve as novel therapeutic targets. However, the substrate specificity of the NSDs and the molecular mechanism of histones $\mathrm{H} 3$ and $\mathrm{H} 4$ recognition and methylation have not yet been established.

Results: Herein, we investigated the in vitro mechanisms of histones $\mathrm{H} 3$ and $\mathrm{H} 4$ recognition and modifications by the catalytic domain of NSD family members. In this study, we quantified in vitro mono-, di- and tri- methylations on H3K4, H3K9, H3K27, H3K36, H3K79, and H4K20 by the carboxyl terminal domain (CTD) of NSD1, NSD2 and NSD3, using histone as substrate. Next, we used a molecular modelling approach and docked 6-mer peptides H3K4 а.a. 1-7; H3K9 а.а. 5-11; H3K27 а.а. 23-29; H3K36 а.а. 32-38; H3K79 а.а. 75-81; H4K20 a.a. 16-22 with the catalytic domain of the NSDs to provide insight into lysine-marks recognition and methylation on histones $\mathrm{H} 3$ and $\mathrm{H} 4$.

Conclusions: Our data highlight the versatility of NSD1, NSD2, and NSD3 for recognizing and methylating several histone lysine marks on histones $\mathrm{H} 3$ and $\mathrm{H} 4$. Our work provides a basis to design selective and specific NSDs inhibitors. We discuss the relevance of our findings for the development of NSD inhibitors amenable for novel chemotherapies.
\end{abstract}

Keywords: Epigenetic therapy of cancer, Histone lysine methyltransferase, NSD1, NSD2/MMSET/WHSC1, NSD3/ WHSC1L, HMTase inhibitors

\section{Background}

Covalent histone modifications are key in chromatin regulatory mechanisms. One such histone modification, lysine methylation, can have both activating and repressive functions on transcriptional events. Histone lysine methyltransferases (HMTases) are transcriptional coregulators that target specific lysines on histones $\mathrm{H} 3$ and $\mathrm{H} 4$, and can transfer up to three methyl groups (Kme1, Kme2, and Kme3) [1]. However, the molecular mechanisms of histone mark recognition remain unclear [2-6]. Epigenetic marks on H3K4, H3K9, H3K27, H3K36, $\mathrm{H} 3 \mathrm{~K} 79$, and H4K20 have been reported to play primary roles in regulating the chromatin and contribute to the histone code that is still obscure [7]. H3K4, H3K36 and

\footnotetext{
* Correspondence: eric.diluccio@gmail.com

Kyungpook National University, School of Applied Biosciences, Life Sciences and Agriculture building \#3, room 309, 80 Daehak-ro, Daegu, Buk-gu 702-701, Republic of Korea
}

H3K79 methylation are associated with a locally relaxed chromatin and transcriptionally active genes, whereas methylation of H3K9, H3K27 and H4K20 are repressors and hallmarks of condensed chromatin at silent loci. Dysfunctions in the regulation of histone methylation are linked to an increasing number of pathological conditions.

The nuclear receptor-binding SET domain (NSD) family, a part of the HMTase KMT3 family, is composed of three HMTases: NSD1, NSD2/MMSET/WHSC1, and NSD3/WHSC1L1 (hereafter NSD1, NSD2 and NSD3) [8]. The NSD proteins are oncogenes highly expressed in numerous pathological conditions and are considered attractive novel therapeutic targets in cancers [2,9-20]. The amplification of NSD1 has been reported in multiple myeloma, lung cancer, neuroblastoma and glioblastoma. The amplification of either NSD1 or NSD2 triggers cellular transformation [21-28]. NSD2 is associated with tumor aggressiveness or prognosis in most types of 
cancers, including prostate cancer and multiple myeloma and is overexpressed in at least 15 different cancers $[9,10,17,18,20,21,25,29-33]$. Increased NSD2 activity is also reported during tumor proliferation in glioblastoma and myeloma [34], resulting in aberrantly high global levels of H3K36me2 [18]. NSD3 is amplified in primary breast carcinoma, bladder cancer, lung cancer, and liver cancer $[12,16,25,35]$. Abnormal fusion proteins containing NSD family members, including NSD1-NUP98 and NSD3-NUP98 fusions, increase H3K36 methylation, leading to acute myeloid leukemia [24-28].

Although NSD proteins are instrumental in the development of numerous cancers, their mechanisms of action in carcinogenesis are still unclear. In particular, the substrate specificity of the NSD members remains unsettled and conflicting data have been reported $[17,20]$. NSD1 has H3K36-specific mono- and di-methylation activities but methylation on H4K20 by NSD1 has also been reported $[3,23,27,36]$. NSD2 activity performs H3K36-specific mono- and di-methylation on chromatin, but di-methylation activity for $\mathrm{H} 4 \mathrm{~K} 20$ at DNA double-strand breaks has also been reported $[17,20]$. A significant increase in H3K36me3 by NSD2 at promoter regions has also recently been demonstrated [4], but potentially ruled-out in another study [20]. In addition, NSD2 has been shown to methylate H3K4, H3K27, and H4K44 [5,6,36-38]. Discrepancies regarding NSD3 methylation activities on H3K4, H3K27, and H3K36 have also been reported [6,24,39-41]. Complicating the mechanisms further, both NSD1 and NSD2 have been shown to methylate NF-kB $[8,42]$. Thus, no consensus on the substrate specificity of the NSD members has been established [17,20,36]. Multiple factors may be responsible for these discrepancies, such as the cellular context, the nature of the substrate (peptide, histone, or nucleosome), the integrity of the enzymes (catalytic domain alone versus full-length protein), and the assays employed.
Despite uncertainties regarding NSD substrates, it appears that increased levels of H3K36 methylation concomitant with decreased levels of H3K27 methylation by deregulated NSD members is a driving mechanism in chromatin regulation [13]. Therefore, the inhibition of NSD members by small molecules represents a valuable therapeutic strategy in several human cancers where elevated levels of NSD members have been reported $[2,4,5,12,14,17,18,26,32,33,43-45]$.

The NSD family members are large multi-domain proteins with four zinc finger domains, two PWWP domains and a catalytic SET domain (Figure 1A) $[9,10,17,20]$. The SET domain is subdivided into pre-SET, SET, and postSET domains, all domains are required for catalytic function (Figure 1A). The function of PHD1-3 and PWWP1-2 domains of the three NSD members on chromatin remains elusive. However, a recent study demonstrated the role of the conserved PHD4 domain in localizing NSD3 on histone H3 [46]. The PHD4 domain of the NSDs may function as a recognition module for histone-lysine marks, helping to position the catalytic SET domain on its lysine substrate [46]. The PHD4 domain is 36-residues downstream of the post-SET subdomain. Due to its immediate vicinity to the post-SET subdomain, PHD4 may be a structural component of the catalytic SET domain. Similarly, the PWWP2 domain is also conserved across NSD members and 72-residues upstream of the pre-SET subdomain. The role of this PWWP2 domain remains unclear.

Previous studies have investigated either full-length NSD members or the catalytic SET domain alone through various experimental approaches, resulting in discrepant conclusions. Herein, we focus on understanding histones $\mathrm{H} 3$ and $\mathrm{H} 4$ recognition and methylation in vitro by the NSDs. In this study, we investigate the function of the carboxyl terminal domain (CTD) of all three NSD members using a consistent experimental approach, in vitro. The NSDs-CTD contains the pre-SET, SET, post-SET, and PHD4 domains within the catalytic core as well as a

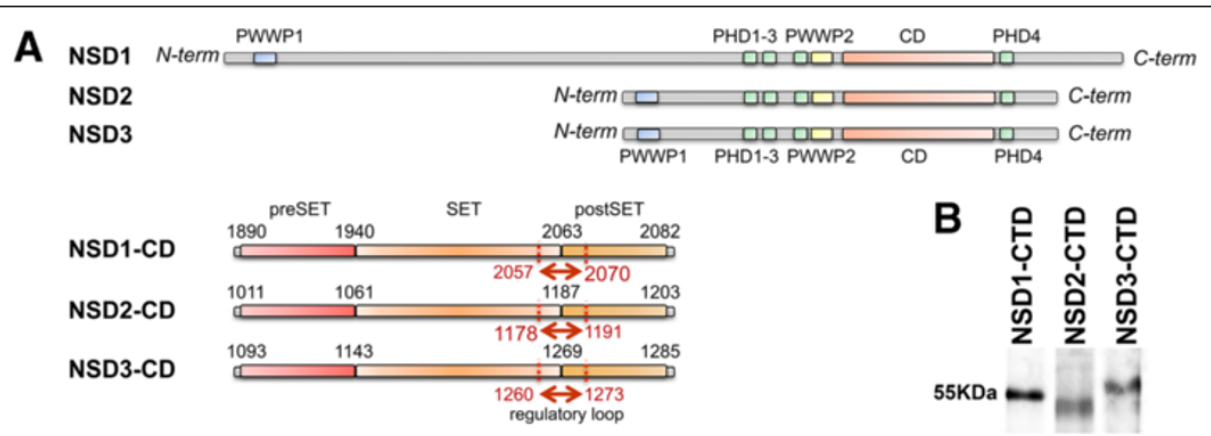

Figure 1 The NSD family of HMTase. A- The schematic primary structure of NSD1, NSD2, and NSD3: All NSD members contain two PWWP domains, four PHD zinc finger domains, and a CD. The CD has HMTase activity and contains the pre-SET, SET, and post-SET domains. The regulatory loop over the histone-binding site is indicated in red. B- Coomassie Blue staining of a SDS-PAGE gel confirms the expressed recombinant NSD1-CTD, NSD2-CTD, and NSD3-CTD. 
histone-recognition module. We quantified the panmethylation properties of the CTD of NSD1, NSD2, and NSD3 on H3K4, H3K9, H3K27, H3К36, H3K79, and H4K20 in vitro. Next, we used MD simulations to elucidate the binding of the NSD catalytic SET domain (CD) on histone tails. Taken together, this study provides insights relevant to the design of specific and selective NSD inhibitors.

\section{Methods}

\section{Cloning}

The carboxyl terminal domains (CTD) of NSD1, NSD2, and NSD3 genes were cloned as follows; NSD1-CTD (1338 bp, 53256663 nt; encoding 446 a.a., 17752221 a.a.), NSD2-CTD (1287 bp, 28084095 nt; encoding 429 a.a., 9361365 a.a.), and NSD3-CTD (1344 bp, 29674311 nt; encoding 484 a.a., 9891437 a.a.) were amplified by PCR using a human liver cDNA library (TAKARA, Japan) as the template. The sets of forward and reverse primers used were $5^{\prime}$-GCTGAGGTCGAC(SalI)CATCC TCGAGCTGTTCCTTCC-3' and 5'-CGGTACGCGG CCGC(NotI)GCACATACTCACGGATCTCCCC-3' for NSD1-CTD, 5' - CAGGCGCATATG(NdeI)TTCCCGT ACATGGAGGGGGAC-3' and 5' - CGCCTGCTCGA G(XhoI)TTTGCCCTCTGTGACTCTCCG-3' for NSD 2-CTD, and 5' - CTGAACGTCGAC(SalI)GGCCTTAA ACATGACTTGGGG-3' and 5' - GACACCGCGGCCG $C(N o t \mathrm{I})$ ATTCTTTTACTTCTTCTCCATG-3' for NSD 3-CTD. The PCR-amplified NSD1-CTD and NSD3CTD fragments (with SalI and NotI restriction sites) and the NSD2-CTD fragment (with NdeI and XhoI restriction sites) were inserted into the corresponding restriction enzyme sites of the multi-cloning site of the protein expression intein-tagging vectors PTYB2 or pTYB12 (New England Biolabs, USA). All sequences were verified by sequencing.

\section{Protein expression and purification}

Escherichia coli expression BL21 (DE3) strain was transformed with pTYB2 or pTYB12 plasmids harbouring NSD1-CTD, NSD2-CTD, and NSD3-CTD (NSD-CTDs). The transformed strains were grown in LB medium containing $100 \mu \mathrm{g} / \mathrm{mL}$ ampicillin and the expression of recombinant NSD-CTDs was induced with $250 \mu \mathrm{M}$ isopropyl 1-thio-D-galactopyranoside (IPTG) for $4 \mathrm{~h}$ at 15 C. E. coli cells were harvested and lysed by a freezethaw method and incubated in buffer A $[20 \mathrm{mM}$ Tris (pH 8.5), $500 \mathrm{mM} \mathrm{NaCl}$ and $0.1 \mathrm{mM}$ EDTA] containing $0.1 \%$ Triton X-100 and $1 \mathrm{mM}$ phenylmethanesulfonylfluoride (PMSF) and treated with 20 cycles of sonication on ice. The resulting cell extract containing NSD-CTDIntein-CBD (chitin-binding domain) fusion proteins was loaded onto an affinity column of chitin beads and washed with 100 column volumes of buffer A with $0.1 \%$ Triton $\mathrm{X}-100$, followed by 20 column volumes of buffer A without Triton X-100. To remove bacterial chaperones bound to the recombinant proteins, the recombinant NSD-CTDbound chitin beads were washed with 10 bed volumes of buffer A containing $10 \mathrm{mM}$ adenosine triphosphate (ATP) and $2.5 \mathrm{mM} \mathrm{MgCl}_{2}$. The affinity column was washed with 20 bed volumes of buffer A and NSD-CTD proteins were cleaved off from the chitin beads by incubation in buffer A with $50 \mathrm{mM} 2$-mercaptoethanol at $4 \mathrm{C}$ for $64 \mathrm{~h}$. After elution in buffer A, samples were concentrated and 2mercaptoethanol was washed off using Amicon Ultra centrifugal filters (Millipore, USA) and then used for methyltransferase assays. A small portion of purified NSD-CTDs was resolved through SDS-PAGE and Coomassie staining showed soluble and pure NSD-CTDs at approximately the expected molecular weight of $52.9 \mathrm{kDa}$ (NSD1-CTD), 49.7 $\mathrm{kDa}$ (NSD2-CTD) and $53.1 \mathrm{kDa}$ (NSD3-CTD) (Figure 1B). The resulting recombinant NSD-CTDs proved stable and retained their catalytic properties for an extended period of time when stored at $-80 \mathrm{C}$.

\section{Histone methyltransferase assays}

Histone methyltransferase activities of NSD-CTDs on H3K4, K9, K27, K36, K79, and H4K20 were measured using colorimetric quantification kits (Epigentek, USA) following the manufacturer s protocol. As for H3K36, H3K79 and H4K20, the purified recombinant NSDCTDs $(0.2 \mu \mathrm{g}$ and $2.0 \mu \mathrm{g})$ were incubated with a recombinant histone $\mathrm{H} 3$ or $\mathrm{H} 4$ (Epigentek, USA) and a methyl group donor (SAM) in the strip wells coated with the specific antibodies for $6090 \mathrm{~min}$ at room temperature. The specific antibodies attached to the bottom of the strip wells captured methylated substrates. As for H3K4, H3K9, and H3K27, instead of the antibody-coated strip wells, biotinylated histone substrates were used and stably captured on the strip wells during the $60 \mathrm{~min}$ incubation at $37 \mathrm{C}$. The captured biotinylated histone substrates were next incubated with a high-affinity antibody for $60 \mathrm{~min}$ at room temperature with shaking at $100 \mathrm{rpm}$. Excess of purified NSD-CTDs, histones, SAM, and antibodies were thoroughly washed away and the strip wells attached with antibody-bound or biotinylated $\mathrm{H} 3 / \mathrm{H} 4 \mathrm{me}$ were incubated with the labeled detection antibodies for $60 \mathrm{~min}$ at $24 \mathrm{C}$, swirling at 100 $\mathrm{rpm}$ in the dark. After thoroughly washing the wells, the methylation level was quantified through a HRPconjugated secondary antibody-color development system with an ELISA plate reader at $450 \mathrm{~nm}$. The antibodies provided by Epigentek are validated and showed no cross-reaction between the substrates. Assays were performed in duplicate. The results were normalized against a control that did not contain any enzymes (NC in Figure 2). 
Molecular modeling, docking, and energy minimization The crystal structure of the catalytic domain (CD) of NSD1 (PDB: 3OOI - a.a. 1850 2080) served as a template for building homology models of NSD2-CD (a.a. 971 1202) and NSD3-CD (a.a. 1044 1283). Multiple-sequence alignment was done with ClustalW V2.0.9 and 100 models were generated using Modeler V9.5. The best model, according to the intrinsic Modeler function, was subjected to sidechains positioning using the SCWRL4 program. Stereochemistry was checked with PROCHECK. The model was manually inspected with COOT. The histone tail peptides were manually built in COOT with the following residues: H3K4 (a.a. 1 7), H3К9 (a.a. 5 11), H3K27 (a.a. 23 29), H3K36 (a.a. 32 38), H3K79 (a.a. 75 81), and H4K20 (a.a. 16 22). A structural overlay between NSD1-CD (PDB: 3OOI) and the crystal structure of SET8 bound to histone H4 peptide (PDB: 3F9Y) was used to manually place and orientate the $\mathrm{H} 3$ - or $\mathrm{H} 4$-peptide tails into the histone binding site of NSD1-CD, NSD2-CD, and NSD3-CD using the SSM algorithm in COOT. Prior to MD calculations, all the raw modelled histone tail peptides exhibited a similar conformation. Electrostatic charge calculations were performed using the PDB2PQR Server (http://nbcr-222. ucsd.edu/pdb2pqr_1.9.0/) with default settings (PARSE forcefield) regardless of the conformation of the peptide tail. We hypothesized the electrostatic charge calculations were not affected by the conformation of the peptide tail as all modelled short peptide tails have a highly similar initial conformation.

Electrostatic calculations were performed with APBS V1.2.1 and the molecular surfaces with the electrostatic properties were rendered with both VMD V1.8.7 and PyMOL V1.2.

\section{Energy minimizations and molecular dynamics simulations in a water box}

The peptide-NSD-CD complexes were fully solvated in a water box (140 100110$)$ using VMD 1.9. The
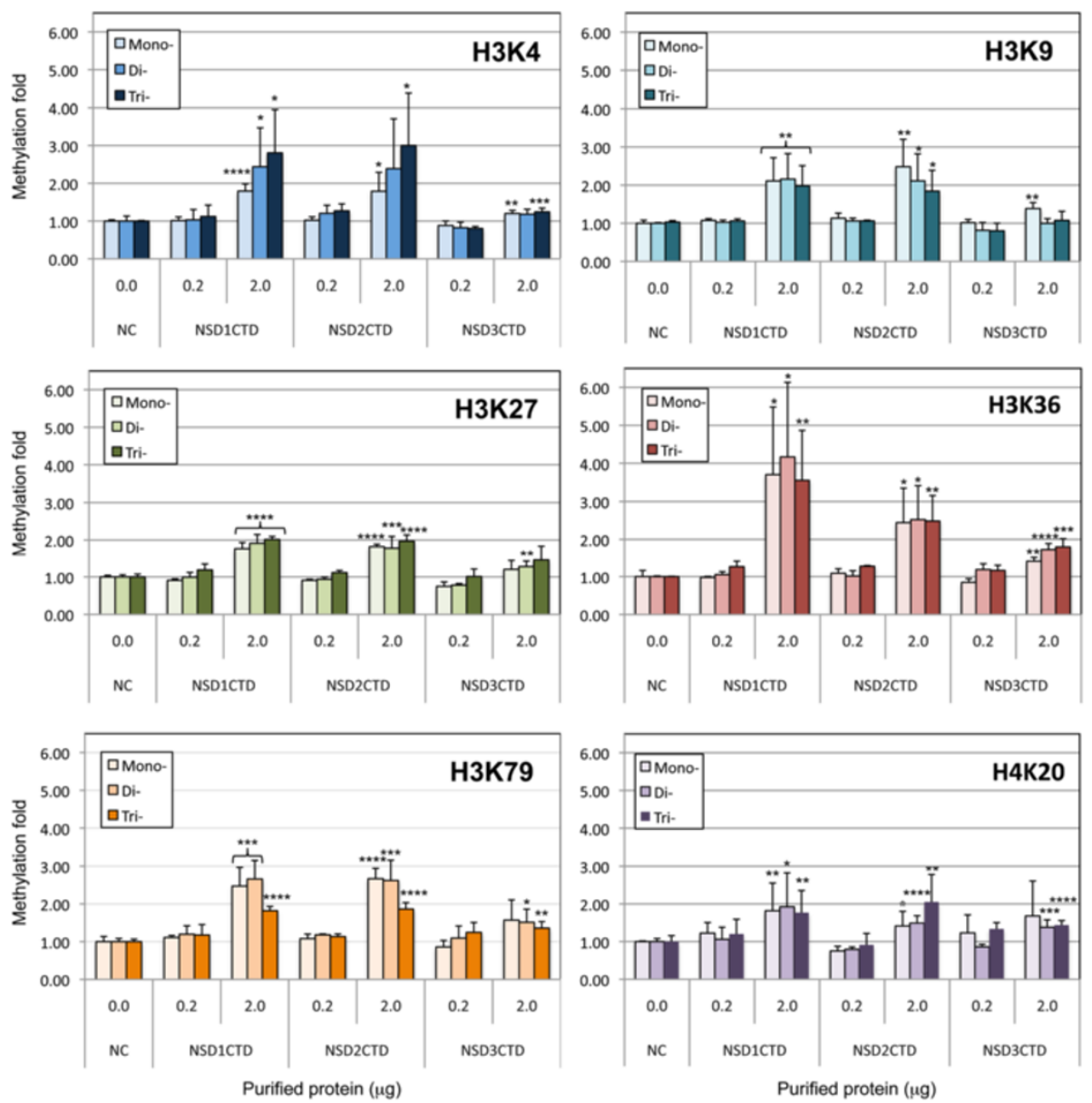

Figure 2 HMTase activities of NSD1-CTD, NSD2-CTD, and NSD3-CTD on H3 and H4 substrates in vitro. HMTase activities of NSD1-CTD, NSD2-CTD, and NSD3-CTD on H3K4, K9, K27, K36, K79, and H4K20 were measured using colorimetric quantification (See Materials and Methods). HMTase assays were done in duplicate individual experiments and all samples were in duplicate. The error bars indicate standard deviations. The results were normalized against controls that did not contain any enzymes (NC, $0 \mu \mathrm{g}$ ). Asterisks indicate P-values; ${ }^{*} \mathrm{p}<0.05,{ }^{* *} \mathrm{p}<0.02,{ }^{* * *} \mathrm{p}<0.005,{ }^{* * * *} \mathrm{p}<0.001$. 
molecular systems used for energy minimizations (EM) and molecular dynamics (MD) contained the following number of atoms: NSD1-CD-H3K4(1 7), 42,121 atoms; NSD1-CD-H3K9(5 11), 42,104 atoms; NSD1-CD-H3K27 (23 27), 42,095 atoms; NSD1-CD-H3K36(32 38), 42,106 atoms; NSD1-CD-H3K79(75 81), 42,120 atoms; NSD1CD-H4K20(32 38), 42,130 atoms; NSD2-CD-H3K4(1 7), 44,848 atoms; NSD2-CD-H3K9(5 11), 44,840 atoms; NSD2-CD-H3K27(23 27), 44,828 atoms; NSD2-CDH3K36(32 38), 44,830 atoms; NSD2-CD-H3K79(75 81), 44,844 atoms; NSD2-CD-H4K20(32 38), 44,860 atoms; NSD3-CD-H3K4(1 7), 44,367 atoms; NSD3CD-H3K9(5 11), 44,353 atoms; NSD3-CD-H3K27(23 27), 44,341 atoms; NSD3-CD-H3K36(32 38), 44,349 atoms; NSD3-CD-H3K79(75 81), 44,366 atoms; NSD3CD-H4K20(32 38), 44,379 atoms. About $90 \%$ of the atoms were from the water molecules. EM and MD were performed with NAMD v2.8 using the CHARMM force field. A constant temperature was maintained using a Langevin damping at $300 \mathrm{~K}$, and the pressure was held constant at 1 atmosphere with a Langevin piston. Electrostatic forces were taken into account using the particle mesh Ewald (PME) method with a 10 cut-off distance. The solvated complexes, histonepeptide-NSD-CD, were first equilibrated with 5,000 steps ( $5 \mathrm{~ns}$ ) of conjugate gradient EM followed by 5 cycles of 20,000 steps (20 ns) of MD and 5,000 steps (5 ns) of EM. The last step was 5 ns of EM. Each solvated complex of histone-peptide-NSD-CD was subjected to a total of 100,000 steps (100 ns) of MD and a total of 30,000 steps (30 ns) of EM. EM steps were long enough to reach a local minimum with $\Delta \varepsilon<1 \mathrm{~kJ} / \mathrm{mol}$. After the MD simulations, the solvated complexes were manually checked for ideal stereochemistry with COOT. The MD trajectories were analyzed with VMD 1.9 and the plots of the total energy versus timescale were plotted using Gnuplot 4.4.

\section{Binding energy determination}

Binding energy was calculated as follows: $\varepsilon_{\text {binding }}=\varepsilon_{\text {com- }}$ plex $\left(\varepsilon_{\text {peptide }}+\varepsilon_{\mathrm{NSD}}\right)$ using the total energy calculations of NAMD [47]. Models of the last MD step were stripped from solvent molecules and used to calculate the binding energy of the complex. Models of the binding complex, peptides, and NSD-CD apo were all subjected to the same EM procedure prior to binding energy calculation. Models were energy minimized with 4,000 steps (4 ns) of conjugate gradient EM followed by 2,000 steps (2 ns) of MD and 6,000 steps (6 ns) of EM (NAMD v2.8) [47]. EM steps were long enough to reach a local minimum with $\Delta \varepsilon<1 \mathrm{~kJ} / \mathrm{mol}$. A constant temperature was maintained by using Langevin damping at $300 \mathrm{~K}$ and the pressure was held constant at 1 atmosphere with a Langevin piston.

\section{Model analysis and validation}

The details of the interactions between the NSD-CD and H3- or H4- peptides were analyzed with Ligplot [48]. The confirmation of interaction maps was manually done through visual inspections of the models in COOT [49].

\section{Results}

The CTD of NSD members can methylate multiple histone lysines in vitro

To examine whether the CTD of NSD1, NSD2, and NSD3 specifically recognizes and methylates multiple lysines of $\mathrm{H} 3$ and $\mathrm{H} 4$ in vitro, we quantified the panmethylation of NSD1-CTD, NSD2-CTD, and NSD3CTD on H3К4, Н3К9, Н3К27, Н3К36, Н3К79, and H4K20 (Figure 2).

Reinberg and colleagues reported that NSD2-SET domain methylates H4K44 on octamers, which could be linked to histone deposition and/or to the response to DNA damage [36]. Although H4K44 methylation remains to be investigated, it was omitted in this study due to lack of a suitable and consistent assay.

Overall, NSD1-CTD, NSD2-CTD, and NSD3-CTD exhibit similar substrate recognition and methylation properties, but reduced methylation activities were observed for NSD3-CTD (Figure 2). H3K4me3 and H3K27me3 species were preferably detected rather than me1 and me2 species for both NSD1-CTD and NSD2-CTD. In contrast, H3K9-me1 \& -me2 and H3K79-me1 \& -me2 species were favored against me3 species.

Unlike NSD1-CTD and NSD2-CTD, NSD3-CTD showed a lower, but significant, activity (up to $\sim 1.5$ fold increase in methylation fold for almost all substrates tested, except for the marginal activity on H3K4 and H3К9) and the methylation properties of NSD3-CTD on H3K9, H3K27, and H3K79 were almost identical to those of NSD1-CTD and NSD2-CTD. However, H4K20 methylation varied among all NSD-CTDs. Nonetheless, significant activity of NSD1-CTD on mono-/tri-methylation, NSD2-CTD on di-/tri-methylation, and NSD3-CTD on di-/tri-methylation of H4K20 were observed, indicating that H4K20 is an in vitro target for all NSD-CTDs. Importantly, H3K36 was methylated by all NSD-CTDs and appears to be the preferable in vitro substrate for NSD1CTD.

Taken together, our data indicate that, in vitro, the CTD of NSD1, NSD2, and NSD3 is able to recognize and methylate H3К4, Н3К9, Н3К27, Н3К36, Н3К79, and $\mathrm{H} 4 \mathrm{~K} 20$ with significant differences in catalytic activities depending on the substrate. Although some HMTases (such as SET9) do not retain significant methyltransferase activity in vitro [50], this was not the case for NSD members, where significant enzymatic activities were measured (Figure 2). 


\section{Molecular modelling and the open-and-closed conformation of the catalytic domain}

Our data showed that, in vitro, the CTD of NSD1, NSD2, and NSD3 has the ability to recognize and methylate several histone lysines (Figure 2). To gain further insight into histone lysine methylation by the NSD members, we investigated conformational modifications of the catalytic domain $(\mathrm{CD})$ to gain access to the lysine-binding pocket. The crystal structure of NSD1-CD (PDB: 3OOI) is devoid of substrate and the histone-lysine binding area is occluded by a regulatory loop at the interface of the SET and post-SET regions (Figure 3) [9,51]. Previously, we showed that molecular determinants of this regulatory loop extend over the histone-binding site, sterically preventing substrates from binding [51-53]. The H3K4 HMTase MLL1 also has been described to have a flexible post-SET responsible for the open-and-closed conformation of the lysine-substrate binding site [53].
The crystal structure of NSD1-CD (PDB: 3OOI - a.a. 1850 2080) was used to build the corresponding CDs of NSD2 and NSD3 by homology modelling. A pair-wise sequence alignment between the template and the target showed that the NSD2-CD (a.a. 971 1202) shares $75.9 \%$ identity and $90.1 \%$ similarity with the NSD1-CD (PDB: 3OO1), whereas the NSD3-CD (a.a. 1044 1283) shares $72.9 \%$ identity and $85 \%$ similarity with the NSD1-CD. Protein sequences sharing at least $40 \%$ identity are likely to share similar structures, thus all NSD1-CD, NSD2-CD, and NSD3-CD are likely to share a similar scaffold (Figure 1).

Next, we used molecular dynamics (MD) to investigate the docking of peptides containing histone lysine (H3K4, H3K9, H3K27, H3K36, H3K79, and H4K20), NSD1-CD, NSD2-CD, and NSD3-CD. The initial models were based on the crystal structure of NSD1-CD solved in a closed apo-conformation. Therefore, models of the apo
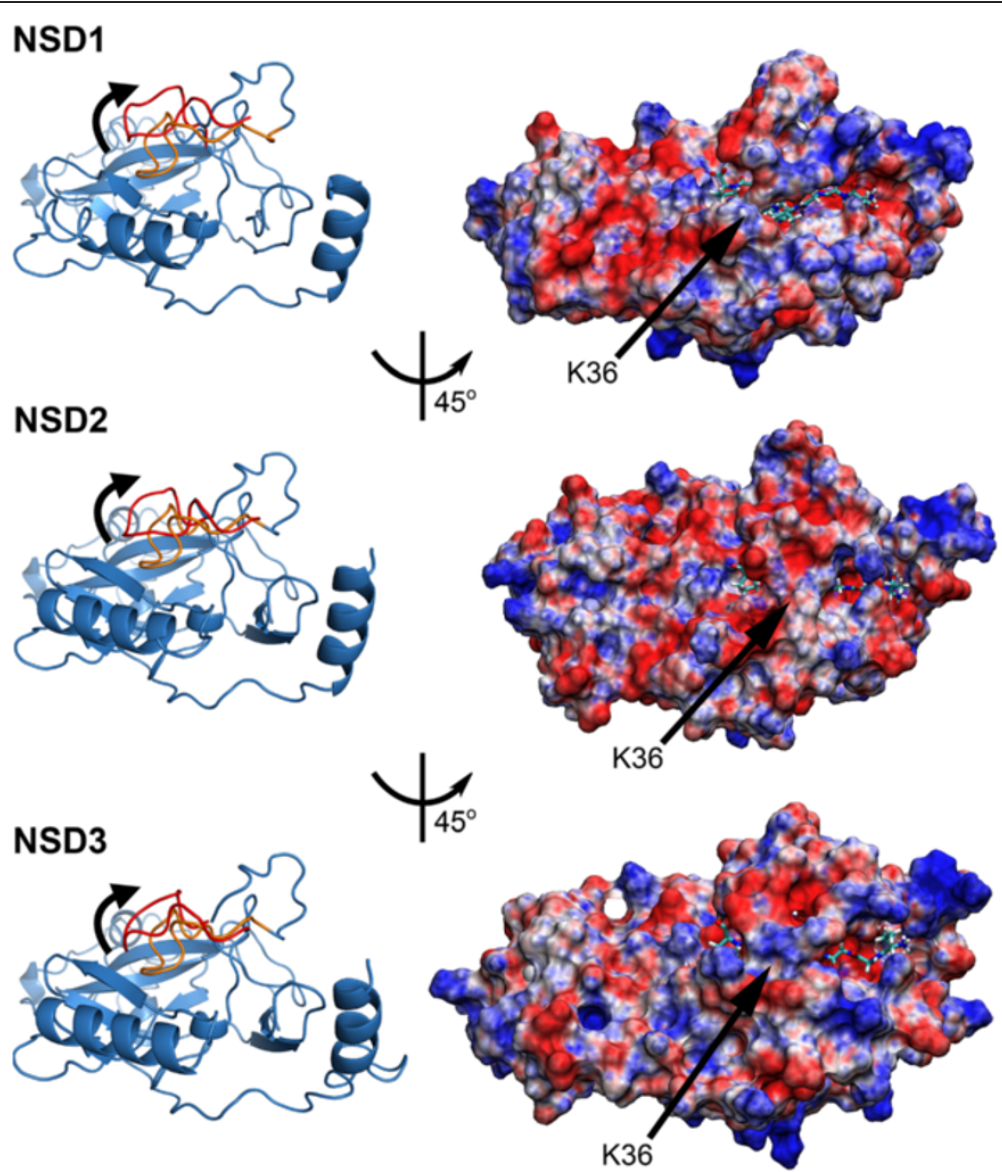

Figure 3 The opening motion of the catalytic domain. Left panel: The structure of the CD is composed of three groups of canonical $\beta$-sheets arranged in a triangular fashion with a group of two $\beta$-sheets closely neighbouring a conserved a-helix defining a cleft for the binding of the lysine-histone ligand. The cofactor SAM binds in a cavity adjacent to the CD connected through a channel. The flexible regulatory loop is shown in red. The motion of the regulatory loop is indicated with bent black closed arrows. Right panel: Model of the NSD-CDs bound with the H3-peptide (a.a. 32 38) after MD simulations. H3K36 is indicated by an arrow. The electrostatic surface is colored as follows: blue: positive charges; red: negative charges with unit $+5 /-5$ kb.T. $e_{c}^{-1}$. 
CD of NSD1, NSD2, and NSD3 docked with peptides were deliberately brought into a sterically unstable conformation. Energy minimizations and long MD simulations performed in a water box centered on the protein relieved the steric clashes and forced the CDs into an open conformation that accommodates substrates (Figures 3, 4, 5 and Additional file 1: Figure S1). Longrange MD simulations were applied to allow complexes to equilibrate into stable conformations with little RMSD oscillations observed for the post-SET regions (overall $\mathrm{c}_{\alpha^{-}}$ RMSD $<0.6$ ) [9]. The resulting models may approximate the conformations of the opened CD potentially resulting from the binding of nucleosomal DNA as an allosteric effector. Following the MD simulations, the structural modifications observed were on the CD of NSD2 and NSD3, specifically on a loop at the interface between the SET and post-SET regions (a.a. 1178 to 1191 for NSD2-CD and a.a. 12601273 for NSD3-CD). This specific loop rotated $\sim 40$ and translated $\sim 6$ at the tip, which is a significant displacement (Figure 3) [9]. The rest of the backbone did not undergo significant structural modifications, with an overall $c_{\alpha}-$ RMSD $<0.6$.

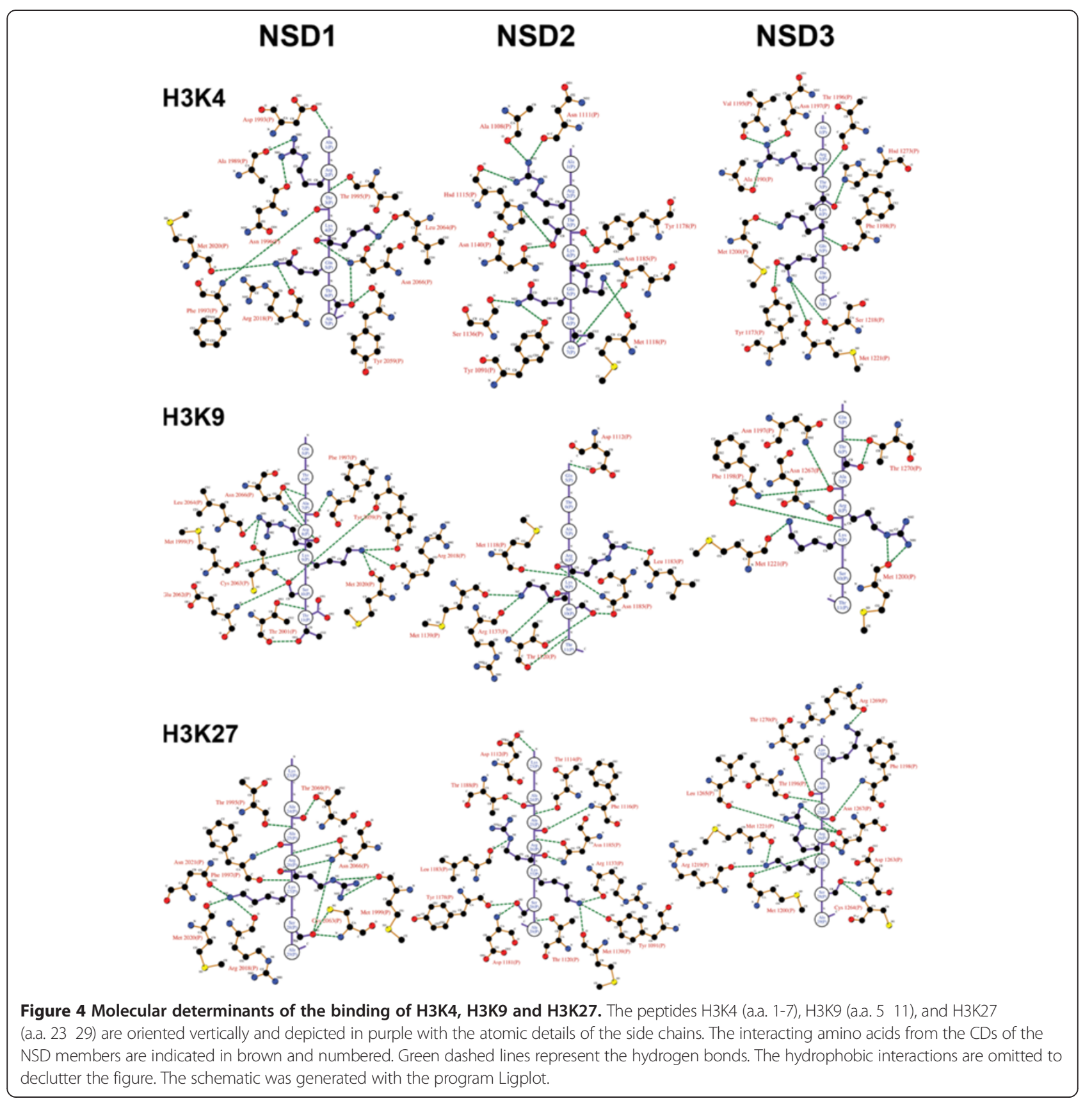




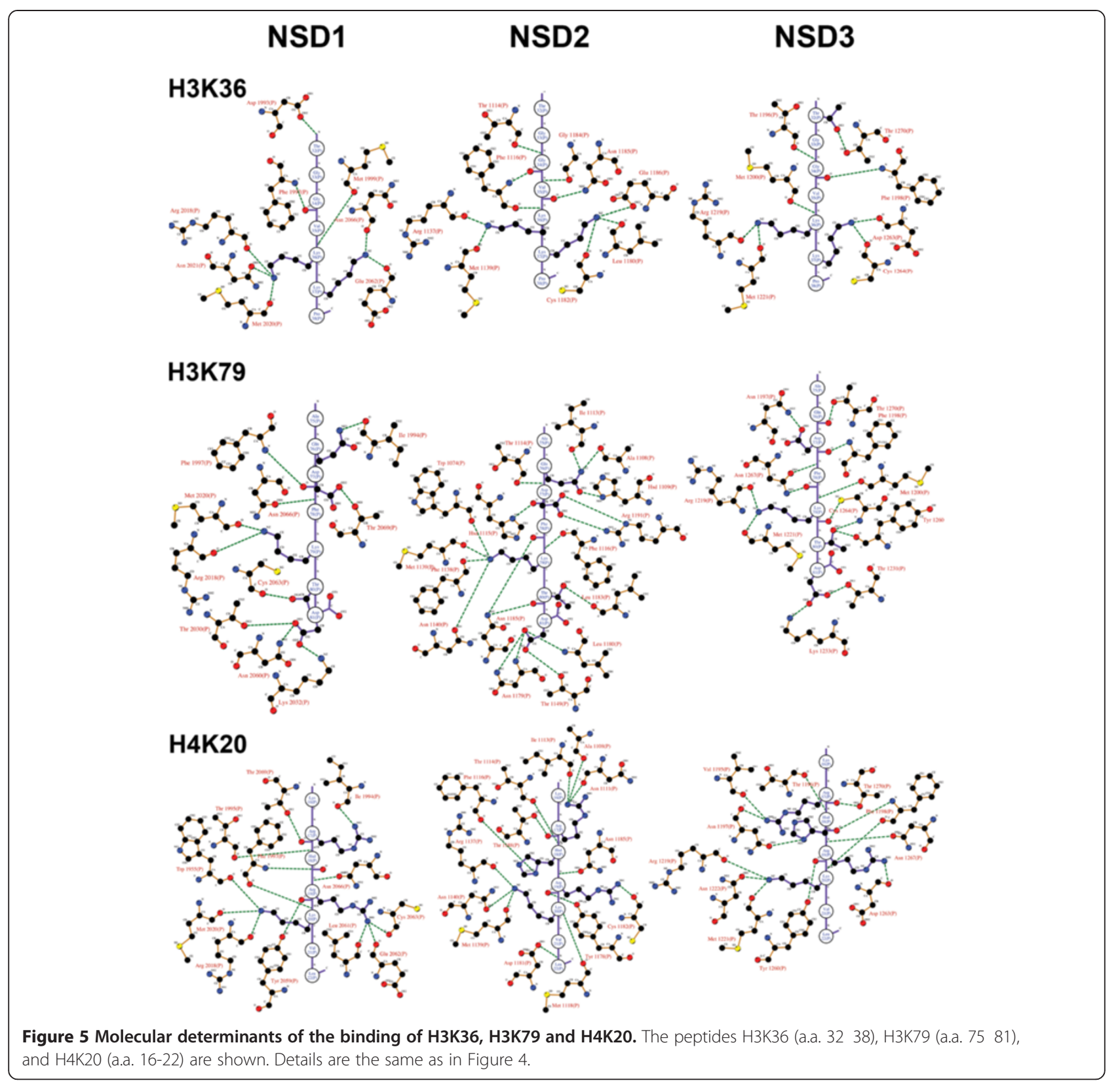

Molecular binding details of H3K4, H3K9, H3K27, H3K36, $\mathrm{H} 3 \mathrm{~K} 79$, and $\mathrm{H} 4 \mathrm{~K} 20$ peptides

The CDs of the NSDs are likely to share highly similar structural features (Figure 3). The histone tail-binding area only becomes accessible when the regulatory loop at the interface between the SET and post-SET regions is rotated and displaced, exposing a negatively-charged surface area suitable for the docking of the positively charged $\mathrm{H} 3$ and $\mathrm{H} 4$ peptide tails (Figure 3 ).

As a step forward towards understanding the molecular details of substrate recognition by NSD-CDs, we used MD simulations to examine the binding characteristics of H3К4, Н3К9, Н3К27, Н3К36, Н3К79, and H4К20 peptides. Overall, our data indicate that all the histone $\mathrm{H} 3$ and $\mathrm{H} 4$ peptides tested (H3K4 a.a. 1-7; H3K9 a.a. 5-11; Н3К27 а.a. 23-29; Н3К36 а.a. 32-38; Н3К79 а.а. 75-81; H4K20 a.a. 16-22) share similar steric hindrance and have similar positive charges related to their electric field and molecular surface. These properties imply favourable binding energies for the NSD-CDs (Table 1).

The total energy of the complexes after the MD simulations was highly comparable between the tested substrates (standard deviation of $0.12 \%$ for both NSD1 and NSD2, and $0.11 \%$ for NSD3), indicating that all peptides tested similarly bind to the NSD members (Table 1). In addition, the regulatory loop acts as a seat belt to anchor a single 
histone tail into the binding domain, which allows the lysine substrate to extend toward the cofactor-binding site for the catalytic reaction to occur (Figure 3). The peptidebinding area is large enough to accommodate a sevenamino acid peptide. Interestingly, the seven-a.a. peptides (containing H3K4, H3K9, H3K27, H3K36, H3K79, and H4K20) share highly similar steric and electrostatic properties (Figures 3, 4 and 5). In addition, all the H3 and H4 histone peptides tested were also firmly stabilized into the histone-tail binding cleft of NSD1, NSD2, and NSD3 by an extensive network of hydrogen bonds (Figures 4 and 5). This observation is consistent with the calculated binding energies, reflecting the proper steric and electrostatic compatibilities between histone peptides and the CDs (Table 1).

The binding of histone $\mathrm{H} 3$ and $\mathrm{H} 4$ peptides induces significant modifications to the histone-binding pocket. In addition to the movement of the regulatory loop seating on top of the histone tail, an induce-fit mechanism triggers changes locally in the backbone along with the rotation of the side chain of key amino acids creating an extensive network of hydrogen bonds anchoring the H3 and H4 peptides (Figures 4 and 5). Specifically, H3K4 (a.a. 1-7) peptide is stabilized by hydrogen bonds with Ala1989, Asp1993, Thr1995, Asn1996, Phe1997, Met2020, Arg2018, Tyr2059, Leu2064 and Asn2066 in NSD1, with Tyr1091, Ala1108, Asn1111, His1115, Met1118, Ser1136, Asn1140, Tyr1178 and Asn1185 in NSD2, and with Tyr1173, Ala1190, Val1195, Thr1196, Asn1197, Phe1198, Met1200, Ser1218, Met1221, and His1273 In NSD3. H3K9 (a.a. 5-11) peptide is stabilized by hydrogen bonds with Phe1997, Thr2001, Arg2018, Met2020, Met1999, Tyr2059, Glu2062, Cys2063, Leu2064 and Asn2066 in NSD1, with Asp1112, Met1118, Thr1120, Arg1137, Met1139, Leu1183 and Asn1185 in NSD2, and with Met1200, Met1221, Asn1267, Asn1197, Phe1198, and Thr1270 in NSD3. H3K27 (a.a. 2329) peptide is stabilized by hydrogen bonds with Thr1995, Phe1997, Met1999, Arg2018, Met2020, Asn2021, Cys2063,

Table 1 Binding energy of peptides containing lysinehistone substrates onto the CD of NSD1, NSD2, and NSD3

\begin{tabular}{lrcr}
\hline (kcal/mol) & NSD1 & NSD2 & NSD3 \\
\hline H3K4 & -229.7 & -299.4 & -200.5 \\
H3K9 & -353.2 & -280.4 & -282.9 \\
H3K27 & -151.9 & -252.6 & -247.4 \\
H3K36 & -277.1 & -272.2 & -261.6 \\
H3K79 & -285.6 & -332.4 & -254.3 \\
H4K20 & -277.2 & -269.5 & -289.0 \\
polyE & 444.2 & 862.9 & 663.2
\end{tabular}

Peptides are as follows: H3K4 (a.a. 1 7); H3K9 (a.a. 5 11); H3K27 (a.a. 23 29); H3K36 (a.a. 32 38); H3K79 (a.a. 75 81); H4K20 (a.a. 16 22). The polyE is a 6-mer poly-glutamic acid peptide used as a control experiment for the docking studies.
Asn2066 and Thr2069 in NSD1, with Tyr1091, Asp1112, Thr1114, Phe1116, Thr1120, Arg1137, Met1139, Tyr1178, Asp1181, Leu1183, Asn1185 and Thr1188 in NSD2, and, with Thr1196, Phe1198, Met1200, Arg1219, Met1221, Asp1263, Cys1264, Leu1265, Asn1267, Arg1269 and Thr1270 in NSD3. H3K36 (a.a. 32-38) peptide is stabilized by hydrogen bonds with Asp1193, Phe1997, Met1999, Arg2018, Met2020, Asn2021, Glu2062 and Asn2066 in NSD1, with Thr1114, Phe1116, Arg1137, Met1139, Leu1180, Cys1182, Gly1184, Asn1185 and Glu1186 in NSD2, and, with Thr1270, Thr1196, Phe1198, Met1200, Arg1219, Met1221, Asp1263 and Cys1264 in NSD3. H3K79 (a.a. 75-81) peptide is stabilized by hydrogen bonds with Ile1994, Phe1997, Arg2018, Met2020, Thr2030, Lys2032, Cys2063, Asn2960, Asn2066 and Thr2069 in NSD1, with Trp1074, Ala1108, His1109, Ile1113, Thr1114, His1115, Phe1116, Phe1138, Met1139, Asn1140, Thr1149, Asn1179, Leu1180, Leu1183, Asn1185, and Arg1191 in NSD2, and with Asn1197, Phe1198, Met1200, Arg1219, Met1221, Thr1231, Lys1233, Tyr1260, Cys1264, Asn1267 and Thr1270 in NSD3. H4K20 (a.a. 16-22) peptide is stabilized by hydrogen bonds with Trp1955, Ile1994, Thr1995, Phe1997, Arg2018, Met2020, Tyr2059, Leu2061, Glu2062, Cys2063, Asn2066 and Thr2069 in NSD1, with Ala1108, Asn1111, Ile1113, Thr1114, Phe1116, Met1118, Arg1137, Met1139, Asn1140, Tyr1178, Asp1181, Cys1182, Asn1185 and Thr1188 in NSD2, and with Val1195, Thr1196, Asn1197, Phe1198, Arg1219, Met1221, Asn1222, Tyr1260, Asp1263, Asn1267 and Thr1270 in NSD3.

The docking of all histone peptides on the $\mathrm{CD}$ is associated with a significantly favorable docking energy, highlighting good substrate compatibilities. As a control experiment, we modelled the docking of a largely electronegative 7-mer poly-glutamic acid (polyE) peptide that induces significant steric hindrance in the peptide binding-pocket. The docking of the polyE peptide is associated with highly unfavorable binding energies, highlighting its poor fit to any of the NSD-CDs, as anticipated (Table 1).

Taken together, our data indicate that the histonebinding pocket of the NSD members is equally compatible with the histone peptides tested, offering steric and electrostatic matches for the stabilization of the $\mathrm{H} 3$ and/ or H4 histone tail(s).

\section{Discussion}

HMTases are essential in maintaining chromatin through modifications of histone tails. In this study, we examined the histone-lysine recognition and methylation properties of NSD1-CTD, NSD2-CTD, and NSD3-CTD in vitro to understand the extent of their catalytic properties and provide medicinal chemistry insights into the design of NSD inhibitors. 
Some HMTases, such as SET9, do not retain significant methyltransferase activity in vitro [50], unlike CTDs of NSD1, NSD2, and NSD3 (Figure 2). Although significant differences in methyltransferase activities have been observed, it is unambiguous that the CTD can recognize and methylate various histone substrates in vitro (Figure 2). Our data indicate that the CTDs of NSD1, NSD2, and NSD3 recognize and methylate H3K4, H3K9, H3K27, $\mathrm{H} 3 \mathrm{~K} 36, \mathrm{H} 3 \mathrm{~K} 79$ and H4K20 in vitro (Figure 2). In addition, other work has shown H4K44 methylation by the NSD2SET domain on octamers [36]. This contrasts with in vivo results where the NSDs are exclusive to one substrate H3K36 or H4K20.

In vivo, NSD2 activity is specific to either H3K36 or H4K20 depending on the cellular context $[17,20]$. The level of HMTase activity also depends on the nature of the substrate (peptide, histone, or nucleosome). The NSD2 SET domain alone can also methylate H3K36 in vitro, generating at least H3K36me1 [36]. H3K36 methylations have been predominantly reported for the NSD members and have significant roles in transcriptional activation, alternative splicing events, and DNA repair [24,54-56]. Methylations on H3K4, H3K9, H3K27, H3K79, and H4K20 by NSD members have also been identified. NSD members might possibly have unique methylation capabilities that respond to a specific cellular context in vivo.

The methylation of non-histone protein targets has previously been shown for HMTases such as G9a targets, WIZ, CDYL1, and ACINUS [57], and SET7/9 target, SUV39H1 [56]. G9a is capable of automethylation on the non-enzymatic N-terminal part of the enzyme [57]. Interestingly, NSD1 and NSD2 methylate both histone and non-histone substrates equally. NF- $\mathrm{kB}$ is activated through NSD1-mediated mono- and di-methylation on lysine 218 [42]. NSD2 directly interacts with NF- $\mathrm{BB}$ for the activation of target genes, including IL-6, IL-8, VEGFA, cyclin D, $\mathrm{Bcl}-2$, and survivin in castration-resistant prostate cancer cells (CRPC) [4]. NSD2 is recruited to the target gene promoters upon induction and mediates NF- $\mathrm{KB}$ activationassociated elevation of histone H3K36me2 and -me3 marks at promoters $[8,42]$. Less is known about NSD3, but non-histone methylation is potentially one of its biological functions as well.

In the absence of substrate, the histone-binding pocket of the NSD members is closed, similar to the H3K9 HMTase MLL1 [53]. Our data suggest that the binding of the histone-peptide itself is not entirely responsible for displacing the post-SET regulatory loop in vivo. Instead, the binding of histones or nucleosomal DNA could be an allosteric effector that triggers the opening of the catalytic domain. Nonetheless, our assays indicate that, in vitro, the binding of either $\mathrm{H} 3$ or $\mathrm{H} 4$ histone triggers the opening of NSD-CD. The binding cavity appears to equally accommodate the 7-mer histone peptides tested (H3K4 a.a. 1-7; H3K9 a.a. 5-11; H3K27 a.a. 2329; Н3К36 а.а. 32-38; Н3К79 а.а. 75-81; H4K20 a.a. 16-22), likely due to all being electrostatically positively charged and presenting homologous steric hindrance. In the case of NSD2, the binding of the H4K20 peptide is favorable and stabilized by an extensive network of hydrogen bonds. The binding of the H3K36 peptide is strongly anchored in the opened-SET domain of NSD2 with ten hydrogen bonds (thirteen for H4K20 a.a. 16-22) (Figure 5). However, a better catalytic efficiency in vitro is observed on H3K36 rather than H4K20, which could perhaps be ascribed to a more favorable conformation of the lysine substrate (Figure 2). Differences calculated in binding energies can be due to differential stabilization properties, which are not necessarily linked with catalytic efficiencies.

Analysis of peptide-protein interactions reveals a conserved binding motif for all the NSD members composed of a quartet of Phenylalanine, Arginine, Methionine and Asparagine (Figures 4 and 5). The trio of conserved Phenylalanine, Arginine and Methionine is located on the pre-SET domain Phe1197 (NSD1), Phe1116 (NSD2), Phe1198 (NSD3); Arg2018 (NSD1), Arg1137 (NSD2), Arg1219 (NSD3); Met2020 (NSD1), Met1139 (NSD2), Met1221 (NSD3) whereas the Asparagine consensus Asn2066 (NSD1), Asn1185 (NSD2) and Asn1267 (NSD3) sits at the interface of the SET and post-SET region, on the flexible regulatory loop we previously identified [9]. In addition, a specific conserved binding motif can be identified for only a pair of NSDs: Met1999 (NSD1) and Met1200 (NSD3); Tyr2059 (NSD1) and Tyr1178 (NSD2); Thr114 (NSD2) and Thr1196 (NSD3). Moreover, peptideprotein interaction specific for each NSD completes the binding motif with Cys2063 (NSD1 specific), Asn1140 (NSD2 specific), Asn1197 \& Asp1263 \& Thr1270 (NSD3 specific). Taken together, the differences observed within the binding motifs amongst the NSDs offer possibilities of discrimination that may be exploited in a drug design strategy targeting the histone tail-binding site.

The post-SET regulatory loop sits on top of the histonepeptide, stabilizing the complex and forcing the lysine substrate into the catalytic channel extending deep toward the co-factor-binding site. In all cases, the lysine substrate extends into the narrow channel connecting the co-factorbinding site in order to react and is anchored by two hydrogen bonds, except for NSD3 with H3K4 and H3K9 where the lysine substrate is bonded by a single hydrogen bond. This double-hydrogen bond is found in other lysine-HMTases and appears to be associated with catalytic efficiency. This observation is consistent with the peptide-bound structures of H3K9-HMTases (GLP and Dim-5) where the Lys9 is stabilized by a pair of analogous hydrogen bonds [52]. A similar conserved doublehydrogen bond is observed in the structures of H4K20- 
SETD8, H3K4-SETD7, and H3K27-vSET [52]. In NSD2, the H3K36 lysine is anchored by two hydrogen bonds whereas the H4K20 lysine is strongly held in place by three hydrogen bonds. In addition, the H3K36 a.a. 32-38 peptide is stabilized by a network of ten hydrogen bonds compared to thirteen for the H4K20 a. a. 16-22 peptide (Figure 5). This observation is consistent with a more favorable binding energy of H4K20 a.a. 16-22 compared to the H3K36 a.a. 32-38 peptide (Table 1). Our data indicate that NSD3-CTD has comparably poor HMTase activities on H3K4 and H3K9, which appear to be correlated with the Lys4 and Lys9 being anchored by only one hydrogen bond. Overall, the stabilization of the lysine substrate is established by a pair of hydrogen bonds that tightly anchor the lysine substrate in the narrow catalytic channel, affecting catalytic efficiency (Figures 2, 3, 4 and 5).

\section{Conclusions}

Our data suggest that the NSD members may not be exclusive to a single histone mark in vivo. We show that the CTDs of NSD members can carry out pan-methylation of lysine substrates and generate K-me1, K-me2, and K-me3 species in vitro. However, in vivo cell-based assays have predominantly identified K-me2 or K-me3 species depending on the cellular context $[2,13,17,18]$. Our molecular modelling data indicate that the binding-groove can equally accommodate K-me2 or K-me3 species. This is consistent with a regulatory mechanism distinct from the $\mathrm{CD}$ and carried out by the PHD4 domain to control the localization and the degree of methylation by NSD members [46]. Interestingly, the PWWP domain of the fission yeast Set9 partner, Pdp1, controls Set9-mediated H4K20 methylation and appears to directly interact with histones for Set9 localization [50]. Moreover, the sequence conservation is high between Pdp1-PWWP and both PWWP domains of NSD1, NSD2, and NSD3 [50]. The hydrophobic residues in Pdp1-PWWP responsible for the recognition of H4K20 marks are also conserved in the two NSDPWWP domains [50]. Like Pdp1, the NSD members may localize to chromatin through the assistance of PWWP domains. Further analysis to elucidate the role of the PWWP domains in the NSD members will greatly improve our understanding of the regulation of histonelysine methylations.

\section{Additional file}

Additional file 1: Standard plots of the total NAMD energy versus timestep during MD simulations. The total energy in $\mathrm{kcal} / \mathrm{mol}$ of the system during the MD simulations is plotted versus time. The plots provide an overview of the five cycles of energy minimization and molecular dynamic simulations.

\section{Abbreviations}

NSD: Nuclear receptor binding SET domain; MMSET: Multiple myeloma SET domain; HMTase: Histone lysine Methyltransferase; SAM: S-adenosylmethionine; MD: Molecular dynamics; RMSD: Root-mean-square deviation.

\section{Competing interests}

The authors declare that they have no competing interests.

\section{Authors contributions}

EDL and MM conceived the project and designed the experiments. EDL, DM, and MM performed experiments. EDL and MM analyzed the data and wrote the manuscript. All authors read and approved the final manuscript.

\section{Authors information}

Website: http://webbuild.knu.ac.kr/ diluccio/.

\section{Acknowledgements}

This study is supported in part by research grants from Astex

Pharmaceuticals (Cambridge, UK) [2014 04260000 and 2014 1440000] and the Basic Science Research Program of the National Research Foundation of Korea (NRF) funded by the Ministry of Education, Science and Technology [2013 08840000]. The Kyungpook National University Research Fund also contributed to the research presented in this study.

Received: 24 July 2014 Accepted: 1 December 2014

Published online: 12 December 2014

\section{References}

1. Krogan NJ, Kim M, Tong A, Golshani A, Cagney G, Canadien V, Richards DP, Beattie BK, Emili A, Boone C, Shilatifard A, Buratowski S, Green Blatt J: Methylation of histone $\mathrm{H} 3$ by Set2 in Saccharomyces cerevisiae is linked to transcriptional elongation by RNA polymerase II. Mol Cell Biol 2003, 23(12):4207 4218.

2. Hudlebusch HR, Santoni-Rugiu E, Simon R, Ralfkiaer E, Rossing HH, Johansen JV, Jorgensen M, Sauter G, Helin K: The histone methyltransferase and putative oncoprotein MMSET is overexpressed in a large variety of human tumors. Clin Cancer Res 2011, 17(9):2919 2933.

3. Berdasco M, Ropero S, Setien F, Fraga MF, Lapunzina P, Losson R, Alaminos M, Cheung NK, Rahman N, Esteller M: Epigenetic inactivation of the Sotos overgrowth syndrome gene histone methyltransferase NSD1 in human neuroblastoma and glioma. Proc Natl Acad Sci U S A 2009, 106(51):21830 21835.

4. Yang P, Guo L, Duan ZJ, Tepper CG, Xue L, Chen X, Kung HJ, Gao AC, Zou $J X$, Chen HW: Histone methyltransferase NSD2/MMSET mediates constitutive NF-kappaB signaling for cancer cell proliferation, survival, and tumor growth via a feed-forward loop. Mol Cell Biol 2012, 32(15):3121 3131.

5. Kim JY, Kee HJ, Choe NW, Kim SM, Eom GH, Baek HJ, Kook H, Seo SB: Multiple-myeloma-related WHSC1/MMSET isoform RE-IIBP is a histone methyltransferase with transcriptional repression activity. Mol Cell Biol 2008, 28(6):2023 2034.

6. Kim SM, Kee HJ, Eom GH, Choe NW, Kim JY, Kim YS, Kim SK, Kook H, Seo SB: Characterization of a novel WHSC1-associated SET domain protein with H3K4 and H3K27 methyltransferase activity. Biochem Biophys Res Commun 2006, 345(1):318 323.

7. Strahl $B D$, Allis $C D$ : The language of covalent histone modifications. Nature 2000, 403(6765):41 45.

8. Zhang X, Wen H, Shi X: Lysine methylation: beyond histones. Acta Biochim Biophys Sin (Shanghai) 2012, 44(1):14 27.

9. Morishita M, di Luccio E: Structural insights into the regulation and the recognition of histone marks by the SET domain of NSD1. Biochem Biophys Res Commun 2011, 412(2):214 219.

10. Morishita M, di Luccio E: Cancers and the NSD family of histone lysine methyltransferases. Biochim Biophys Acta 2011, 1816(2):158 163.

11. Mirabella F, Wu P, Wardell CP, Kaiser MF, Walker BA, Johnson DC, Morgan $\mathrm{GJ}$ : MMSET is the key molecular target in $\mathrm{t}(4 ; 14)$ myeloma. Blood Cancer J 2013, 3:e114.

12. Kang D, Cho HS, Toyokawa G, Kogure M, Yamane Y, Iwai Y, Hayami S, Tsunoda T, Field HI, Matsuda K, Neal DE, Ponder BA, Maehara Y, Nakamura $Y$, Hamamoto R: The histone methyltransferase Wolf-Hirschhorn syndrome candidate 1-like 1 (WHSC1L1) is involved in human carcinogenesis. Genes Chromosomes Cancer 2013, 52(2):126 139. 
13. Wagner EJ, Carpenter PB: Understanding the language of Lys36 methylation at histone H3. Nat Rev Mol Cell Biol 2012, 13(2):115 126.

14. Ezponda T, Popovic R, Shah MY, Martinez-Garcia E, Zheng Y, Min DJ, Will C, Neri A, Kelleher NL, YU J, Licht JD: The histone methyltransferase MMSET/WHSC1 activates TWIST1 to promote an epithelial-mesenchymal transition and invasive properties of prostate cancer. Oncogene 2012, 32(23):2882 2890

15. Varier RA, Timmers HT: Histone lysine methylation and demethylation pathways in cancer. Biochim Biophys Acta 2011, 1815(1):75 89.

16. Toyokawa G, Cho HS, Masuda K, Yamane Y, Yoshimatsu M, Hayami S, Takawa M, Iwai Y, Daigo Y, Tsuchiya E, Tsunoda T, Field HI, Kelly JD, Neal DE, Maehara Y, Ponder BA, Nakamura Y, Hamamoto R: Histone lysine methyltransferase Wolf-Hirschhorn syndrome candidate 1 is involved in human carcinogenesis through regulation of the Wnt pathway. Neoplasia 2011, 13(10):887 898.

17. Pei H, Zhang L, Luo K, Qin Y, Chesi M, Fei F, Bergsagel PL, Wang L, You Z, Lou Z: MMSET regulates histone H4K20 methylation and 53BP1 accumulation at DNA damage sites. Nature 2011, 470(7332):124 128

18. Martinez-Garcia E, Popovic R, Min DJ, Sweet SM, Thomas PM, Zamdborg L, Heffner A, Will C, Lamy L, Staudt LM, Levens DL, Kelleher NL, Licht JD: The MMSET histone methyl transferase switches global histone methylation and alters gene expression in $\mathrm{t}(4 ; 14)$ multiple myeloma cells. Blood 2011 117(1):211 220.

19. Kurdistani SK: Histone modifications in cancer biology and prognosis. Prog Drug Res 2011, 67:91 106

20. Kuo AJ, Cheung P, Chen K, Zee BM, Kioi M, Lauring J, Xi Y, Park BH, Shi X, Garcia BA, Li W, Gozani O: NSD2 links dimethylation of histone H3 at lysine 36 to oncogenic programming. Mol Cell 2011, 44(4):609 620

21. Schneider R, Bannister AJ, Kouzarides T: Unsafe SETs: histone lysine methyltransferases and cancer. Trends Biochem Sci 2002, 27(8):396 402.

22. Zhao F, Chen Y, Li R, Zeng R, Wen L, Liu Y, Zhang C: Role of triptolide in cell proliferation, cell cycle arrest, apoptosis and histone methylation in multiple myeloma U266 cells. Eur J Pharmacol 2010, 646(1-3):1 11.

23. Lucio-Eterovic AK, Singh MM, Gardner JE, Veerappan CS, Rice JC, Carpenter $\mathrm{PB}$ : Role for the nuclear receptor-binding SET domain protein 1 (NSD1) methyltransferase in coordinating lysine 36 methylation at histone 3 with RNA polymerase II function. Proc Natl Acad Sci U S A 2010, 107(39):16952 16957.

24. Wang GG, Cai L, Pasillas MP, Kamps MP: NUP98-NSD1 links H3K36 methylation to Hox-A gene activation and leukaemogenesis. Nat Cell Biol 2007, 9(7):804 812

25. Rosati R, La Starza R, Veronese A, Aventin A, Schwienbacher C, Vallespi T, Negrini M, Martelli MF, Mecucci C: NUP98 is fused to the NSD3 gene in acute myeloid leukemia associated with $\mathrm{t}(8 ; 11)(\mathrm{p} 11.2 ; \mathrm{p} 15)$. Blood 2002, 99(10):3857 3860 .

26. Marango J, Shimoyama M, Nishio H, Meyer JA, Min D-J, Sirulnik A, MartinezMartinez Y, Chesi M, Bergsagel PL, Zhou M-M, Waxman S, Leibovitch BA, Walsh MJ, Licht JD: The MMSET protein is a histone methyltransferase with characteristics of a transcriptional corepressor. Blood 2008, 111(6):3145 3154

27. Rayasam GV, Wendling O, Angrand P-O, Mark M, Niederreither K, Song L, Lerouge T, Hager GL, Chambon P, Losson R: NSD1 is essential for early post-implantation development and has a catalytically active SET domain. Embo J 2003, 22(12):3153 3163

28. Walter MJ, Payton JE, Ries RE, Shannon WD, Deshmukh H, Zhao Y, Baty J, Heath S, Westervelt P, Watson MA, Tomasson MH, Nagarajan R, O Gara BP, Bloomfield CD, Mrzek K, Selzer RR, Richmond TA, Kitzman J, Geoghegan J, Eis PS, Maupin R, Fulton RS, McLellan M, Wilson RK, Mardis ER, Link DC, Graubert TA, DiPersio JF, Ley TJ: Acquired copy number alterations in adult acute myeloid leukemia genomes. Proc Natl Acad Sci U S A 2009, 106(31):12950 12955.

29. Lucio-Eterovic A: An open and shut case for the role of NSD proteins as oncogenes. Transcription 2011, 2(4):158 161

30. Strahl BD, Grant PA, Briggs SD, Sun Z-W, Bone JR, Caldwell JA, Mollah S, Cook RG, Shabanowitz J, Hunt DF, Allis CD: Set2 Is a Nucleosomal Histone H3-Selective Methyltransferase That Mediates Transcriptional Repression. Mol Cell Biol 2002, 22(5):1298 1306.

31. Kang HB, Choi Y, Lee JM, Choi KC, Kim HC, Yoo JY, Lee YH, Yoon HG: The histone methyltransferase, NSD2, enhances androgen receptor-mediated transcription. FEBS Lett 2009, 583(12):1880 1886.

32. Kassambara A, Klein B, Moreaux J: MMSET is overexpressed in cancers: Link with tumor aggressiveness. Biochem Biophys Res Commun 2009, 379(4):840 845 .
33. Brito JL, Walker B, Jenner M, Dickens NJ, Brown NJ, Ross FM, Avramidou A Irving JA, Gonzalez D, Davies FE, Morgan GJ: MMSET deregulation affects cell cycle progression and adhesion regulons in $\mathrm{t}(4 ; 14)$ myeloma plasma cells. Haematologica 2009, 94(1):78 86.

34. Li J, Yin C, Okamoto H, Mushlin H, Balgley BM, Lee CS, Yuan K, Ikejiri B, Glasker S, Vortmeyer AO, Oldfield EH, Weil RJ, Zhuang Z: Identification of a novel proliferation-related protein, WHSC1 4a, in human gliomas. Neuro Oncol 2008, 10(1):45 51.

35. Angrand PO, Apiou F, Stewart AF, Dutrillaux B, Losson R, Chambon P: NSD3, a new SET domain-containing gene, maps to $8 \mathrm{p} 12$ and is amplified in human breast cancer cell lines. Genomics 2001, 74(1):79 88.

36. Li Y, Trojer P, Xu CF, Cheung P, Kuo A, Drury WJ 3rd, Qiao Q, Neubert TA, Xu RM, Gozani O, Reinberg D: The target of the NSD family of histone lysine methyltransferases depends on the nature of the substrate. J Biol Chem 2009, 284(49):34283 34295.

37. Margueron R, Justin N, Ohno K, Sharpe ML, Son J, Drury WJ 3rd, Voigt P, Martin SR, Taylor WR, De Marco V, Pirrotta V, Reinberg D, Gamblin SJ: Role of the polycomb protein EED in the propagation of repressive histone marks. Nature 2009, 461(7265):762 767.

38. Nimura K, Ura K, Shiratori H, Ikawa M, Okabe M, Schwartz RJ, Kaneda Y: A histone $\mathrm{H} 3$ lysine 36 trimethyltransferase links Nkx2-5 to WolfHirschhorn syndrome. Nature 2009, 460(7252):287 291.

39. Zhou Z, Thomsen R, Kahns S, Nielsen AL: The NSD3L histone methyltransferase regulates cell cycle and cell invasion in breast cancer cells. Biochem Biophys Res Commun 2010, 398(3):565 570

40. Fang R, Barbera AJ, Xu Y, Rutenberg M, Leonor T, Bi Q, Lan F, Mei P, Yuan GC, Lian C, Peng J, Cheng D, Sui G, Kaiser UB, Shi Y, Shi YG: Human LSD2/KDM1b/AOF1 regulates gene transcription by modulating intragenic H3K4me2 methylation. Mol Cell 2010, 39(2):222 233.

41. Taketani T, Taki T, Nakamura H, Taniwaki M, Masuda J, Hayashi Y: NUP98NSD3 fusion gene in radiation-associated myelodysplastic syndrome with $\mathrm{t}(8 ; 11)(\mathrm{p} 11 ; \mathrm{p} 15)$ and expression pattern of NSD family genes. Cancer Genet Cytogenet 2009, 190(2):108 112.

42. Lu T, Jackson MW, Wang B, Yang M, Chance MR, Miyagi M, Gudkov AV, Stark GR: Regulation of NF-kappaB by NSD1/FBXL11-dependent reversible lysine methylation of p65. Proc Natl Acad Sci U S A 2010, 107(1):46 51.

43. Asangani IA, Ateeq B, Cao Q, Dodson L, Pandhi M, Kunju LP, Mehra R, Lonigro RJ, Siddiqui J, Palanisamy N, Wu YM, Cao X, Kim JH, Zhao M, Qin ZS, lyer MK, Maher CA, Kumar-Sinha C, Varambally S, Chinnaiyan AM: Characterization of the EZH2-MMSET Histone Methyltransferase Regulatory Axis in Cancer. Mol Cell 2012, 49(1):80 93.

44. Chesi M, Nardini E, Lim RS, Smith KD, Kuehl WM, Bergsagel PL: The t(4;14) translocation in myeloma dysregulates both FGFR3 and a novel gene, MMSET, resulting in IgH/MMSET hybrid transcripts. Blood 1998, 92(9):3025 3034

45. Xie Z, Chng WJ: MMSET: role and therapeutic opportunities in multiple myeloma. BioMed research international 2014, 2014:636514.

46. He C, Li F, Zhang J, Wu J, Shi Y: The methyltransferase NSD3 has chromatin-binding motifs, PHD5- $\mathrm{C} 5 \mathrm{HCH}$, that are distinct from other NSD (nuclear receptor SET domain) family members in their histone H3 recognition. J Biol Chem 2013, 288(7):4692 4703.

47. Phillips JC, Braun R, Wang W, Gumbart J, Tajkhorshid E, Villa E, Chipot C, Skeel RD, Kale L, Schulten K: Scalable molecular dynamics with NAMD. J Comput Chem 2005, 26(16):1781 1802.

48. Wallace AC, Laskowski RA, Thornton JM: LIGPLOT: a program to generate schematic diagrams of protein-ligand interactions. Protein Eng 1995, 8(2):127 134.

49. Emsley P, Cowtan K: Coot: model-building tools for molecular graphics. Acta Crystallogr D Biol Crystallogr 2004, 60(Pt 12 Pt 1):2126 2132.

50. Wang Y, Reddy B, Thompson J, Wang H, Noma K, Yates JR 3rd, Jia S: Regulation of Set9-mediated H4K20 methylation by a PWWP domain protein. Mol Cell 2009, 33(4):428 437.

51. Qiao Q, Li Y, Chen Z, Wang M, Reinberg D, Xu RM: The structure of NSD1 reveals an autoregulatory mechanism underlying histone $\mathrm{H} 3 \mathrm{~K} 36$ methylation. J Biol Chem 2011, 286(10):8361 8368.

52. Wu H, Min J, Lunin W, Antoshenko T, Dombrovski L, Zeng H, Allali-Hassani A Campagna-Slater V, Vedadi M, Arrowsmith CH, Plotnikov AN, Schapira M: Structural biology of human H3K9 methyltransferases. PLoS One 2010, 5(1):e8570.

53. Southall SM, Wong PS, Odho Z, Roe SM, Wilson JR: Structural basis for the requirement of additional factors for MLL1 SET domain activity and recognition of epigenetic marks. Mol Cell 2009, 33(2):181 191. 
54. Morris SA, Shibata Y, Noma K, Tsukamoto Y, Warren E, Temple B, Grewal SIS, Strahl BD: Histone H3K36 methylation is associated with transcription elongation in Schizosaccharomyces pombe. Eukaryotic Cell 2005, 4(8):1446 1454.

55. Abu-Farha M, Lambert JP, Al-Madhoun AS, Elisma F, Skerjanc IS, Figeys D: The tale of two domains: proteomics and genomics analysis of SMYD2, a new histone methyltransferase. Mol Cell Proteomics 2008, 7(3):560 572.

56. Wang D, Zhou J, Liu X, Lu D, Shen C, Du Y, Wei FZ, Song B, Lu X, Yu Y, Wang L, Zhao Y, Wang H, Yang Y, Akiyama Y, Zhang H, Zhu WG: Methylation of SUV39H1 by SET7/9 results in heterochromatin relaxation and genome instability. Proc Natl Acad Sci U S A 2013, 110(14):5516 5521.

57. Rathert P, Dhayalan A, Murakami M, Zhang X, Tamas R, Jurkowska R, Komatsu Y, Shinkai Y, Cheng XD, Jeltsch A: Protein lysine methyltransferase G9a acts on non-histone targets. Nat Chem Biol 2008, 4(6):344 346.

doi:10.1186/s12900-014-0025-x

Cite this article as: Morishita et al:: In vitro histone lysine methylation by NSD1, NSD2/MMSET/WHSC1 and NSD3/WHSC1L. BMC Structural Biology 2014 14:25

\section{Submit your next manuscript to BioMed Central and take full advantage of:}

QConvenient online submission

$\otimes$ Thorough peer review

$\nabla$ No space constraints or color $\nabla$ gure charges

$\nabla$ Immediate publication on acceptance

Q Inclusion in PubMed, CAS, Scopus and Google Scholar

$\otimes$ Research which is freely available for redistribution 